\title{
Compresión del tronco de la arteria coronaria izquierda por la dilatación de la arteria pulmonar principal en pacientes con hipertensión pulmonar: tratamiento y seguimiento a largo plazo
}

\section{Left main coronary artery compression due to dilatation of the main pulmonary artery in patients with pulmonary hypertension: treatment and long-term follow-up}

\author{
Juan M. Farina*, Guillermina Sorasio, Víctor Darú y Adrián J. Lescano \\ Servicio de Cardiología, Red de Clínicas Santa Clara. Buenos Aires, Argentina
}

Se describe el caso de una paciente de sexo femenino, de 56 años de edad, con diagnóstico de hipertensión pulmonar idiopática tres años antes de la consulta, quien a su ingreso refirió disnea y dolor precordial de características anginosas en clase funcional III de dos semanas de evolución. Al ingreso se confirmaron signos clínicos de insuficiencia cardíaca derecha (edemas en miembros inferiores, leve derrame pleural bilateral, reflujo hepatoyugular positivo), aumento de la intensidad del segundo ruido cardíaco a expensas del componente pulmonar y soplo expulsivo en foco pulmonar. El estudio diagnóstico inicial incluyó un electrocardiograma con hipertrofia ventricular derecha y bloqueo completo de rama derecha. El ecocardiograma Doppler evidenció dilatación de cámaras derechas con depresión leve de la función sistólica del ventrículo derecho y una insuficiencia tricuspídea moderada con una velocidad pico de $4.52 \mathrm{~m} / \mathrm{s}$, una presión sistólica de la arteria pulmonar calculada de $90 \mathrm{mmHg}$ (con base en los $82 \mathrm{mmHg}$ de gradiente entre ventrículo y aurícula derecha sumados a los $8 \mathrm{mmHg}$ de presión en la aurícula derecha determinados a partir del diámetro y colapso de la vena cava inferior). De manera adicional, este método posibilitó la sospecha de la compresión extrínseca del tronco de la coronaria izquierda como causa de angina al hallar una dilatación grave del tronco de la arteria pulmonar ( $55 \mathrm{~mm}$ ), así como de sus ramas principales ${ }^{1}$. Ante dicha sospecha se indicó una angiotomografía de tórax que confirmó la marcada dilatación de la arteria pulmonar (54 mm en este caso por este método) y delineó el trayecto anormal del tronco de la coronaria izquierda, con marcada reducción de su luz, disminución de la distancia entre éste y la arteria pulmonar y con la formación de un ángulo muy estrecho entre él y el seno de Valsalva izquierdo (Fig. 1A) ${ }^{2}$. Tras asumir la compresión extrínseca por parte de la arteria pulmonar, se realizó con fines diagnósticos iniciales y potencialmente terapéuticos una coronariografía que mostró obstrucción grave del tronco de la coronaria izquierda a nivel ostial, con flujo de la escala de TIMI (thrombolysis in myocardial infarction) 3 y un trayecto casi paralelo a la pared del seno de Valsalva izquierdo por el desplazamiento generado por la compresión (Fig. 1B). Para una mejor definición de la lesión se utilizó ultrasonido intravascular durante el procedimiento, que registró la ausencia de placas de ateroma y confirmó la deformación de la luz de perfil elíptico, la reducción crítica del diámetro arterial a nivel ostial y la naturaleza puramente extrínseca de la obstrucción (Fig. 1C) ${ }^{3}$. A continuación se decidió
1405-9940/○ 2020 Instituto Nacional de Cardiología Ignacio Chávez. Publicado por Permanyer. Este es un artículo open access bajo la licencia CC
Correspondencia:

*Juan M. Farina

E-mail: juan.farina@gmail.com

DOI: 10.24875/ACM.20000319 BY-NC-ND (http://creativecommons.org/licenses/by-nc-nd/4.0/). 


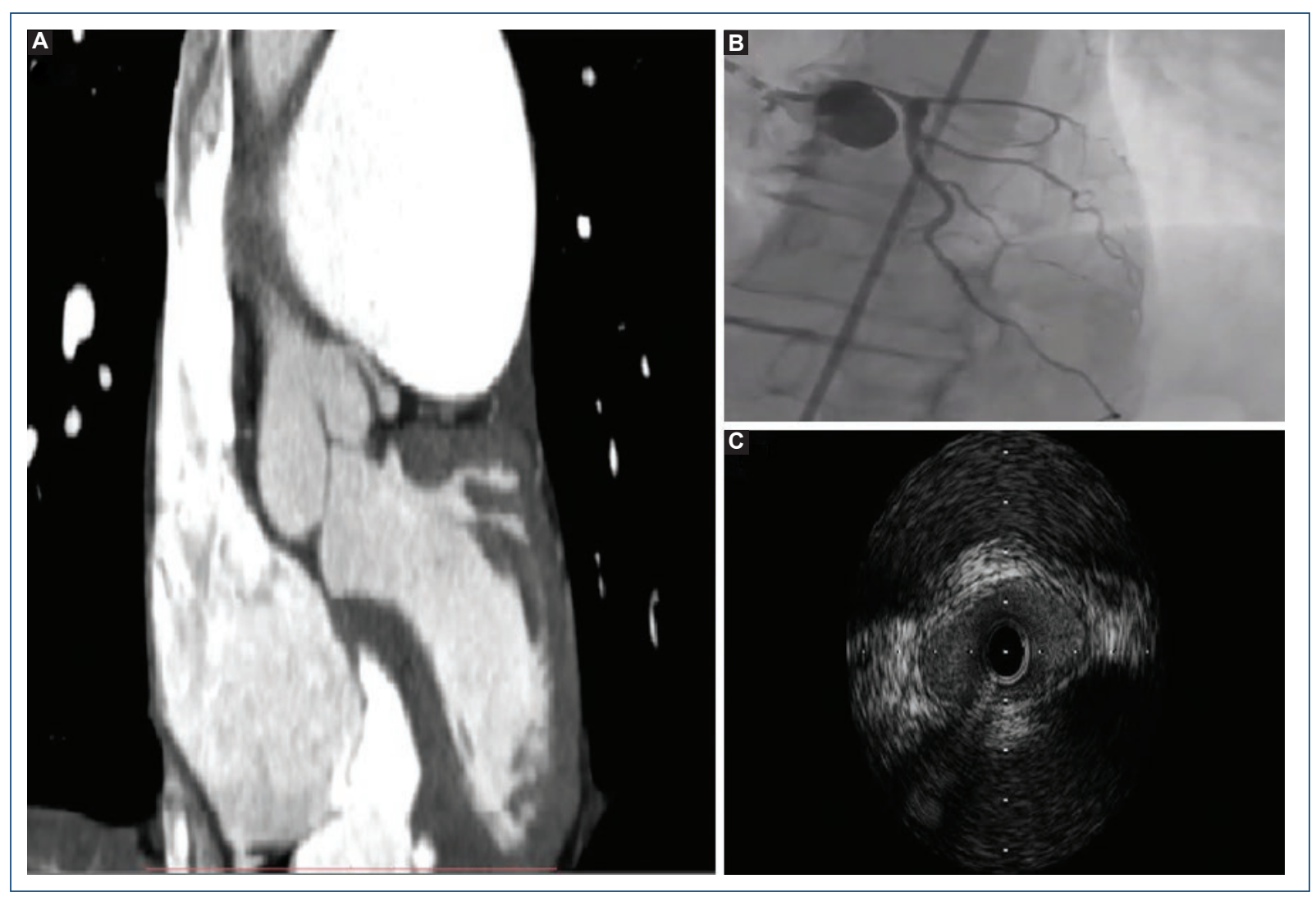

Figura 1. A: angiotomografía de tórax, reconstrucción coronal: marcada dilatación de la arteria pulmonar con estrecho espacio entre ésta y el seno de Valsalva, lo que modifica el trayecto del tronco de la coronaria izquierda. Nótese el ángulo muy agudo entre el seno de Valsalva y el tronco de la coronaria izquierda, con reducción de la luz de este último. B: coronariografía: grave estrechez del tronco de la coronaria izquierda desde su nacimiento. El resto del árbol coronario izquierdo no muestra lesiones significativas. C: ultrasonido intravascular: se descarta la presencia de placas ateroescleróticas. Se observa la deformación de la luz del tronco de la coronaria izquierda que se torna inicialmente elíptica por compresión hasta hacerse mínima $\left(2 \mathrm{~mm}^{2}\right)$.

el implante de un stent liberador de fármaco de 4.5 x $20 \mathrm{~mm}$ en el tronco de la coronaria izquierda, sin complicaciones. La paciente evolucionó de modo favorable y se realizó un seguimiento anual con angiotomografía de tórax sin evidencias de complicaciones a tres años del implante.

En pacientes con diagnóstico de hipertensión pulmonar, la angina de pecho es un síntoma de frecuente aparición, referido por el 35 a $40 \%$ de todos los pacientes con esta enfermedad ${ }^{4}$. Su aparición se ha atribuido por lo general al desequilibrio metabólico generado por la hipertrofia y la sobrecarga del ventrículo derecho más que al compromiso coronario ${ }^{5}$. Sin embargo, la compresión extrínseca del tronco de la arteria coronaria izquierda por la dilatación de la arteria pulmonar principal es una entidad cada vez más reconocida como causa de este síntoma, con un prevalencia notificada cercana al $40 \%$ en pacientes portadores de hipertensión pulmonar que refieren angina o equivalentes anginosos ${ }^{6}$. Esta complicación mecánica es tratable, pero en ausencia de resolución puede tener consecuencias graves como el infarto agudo de miocardio, la disfunción ventricular izquierda, las arritmias cardíacas, e incluso la muerte súbita, por lo que es una entidad de interés clínico relevante ${ }^{7}$. Con respecto al tratamiento, las alternativas posibles son la operación de revascularización miocárdica y la angioplastia percutánea. Si se considera el alto riesgo quirúrgico que presentan habitualmente los pacientes portadores de hipertensión pulmonar, hoy en día se prefiere la revascularización a través de procedimientos percutáneos, con la colocación de stents ${ }^{8}$. No existe evidencia clara sobre cuál es el tipo de stent que debe utilizarse (convencional o liberador de fármaco), por lo que la decisión debe tomarse con base 


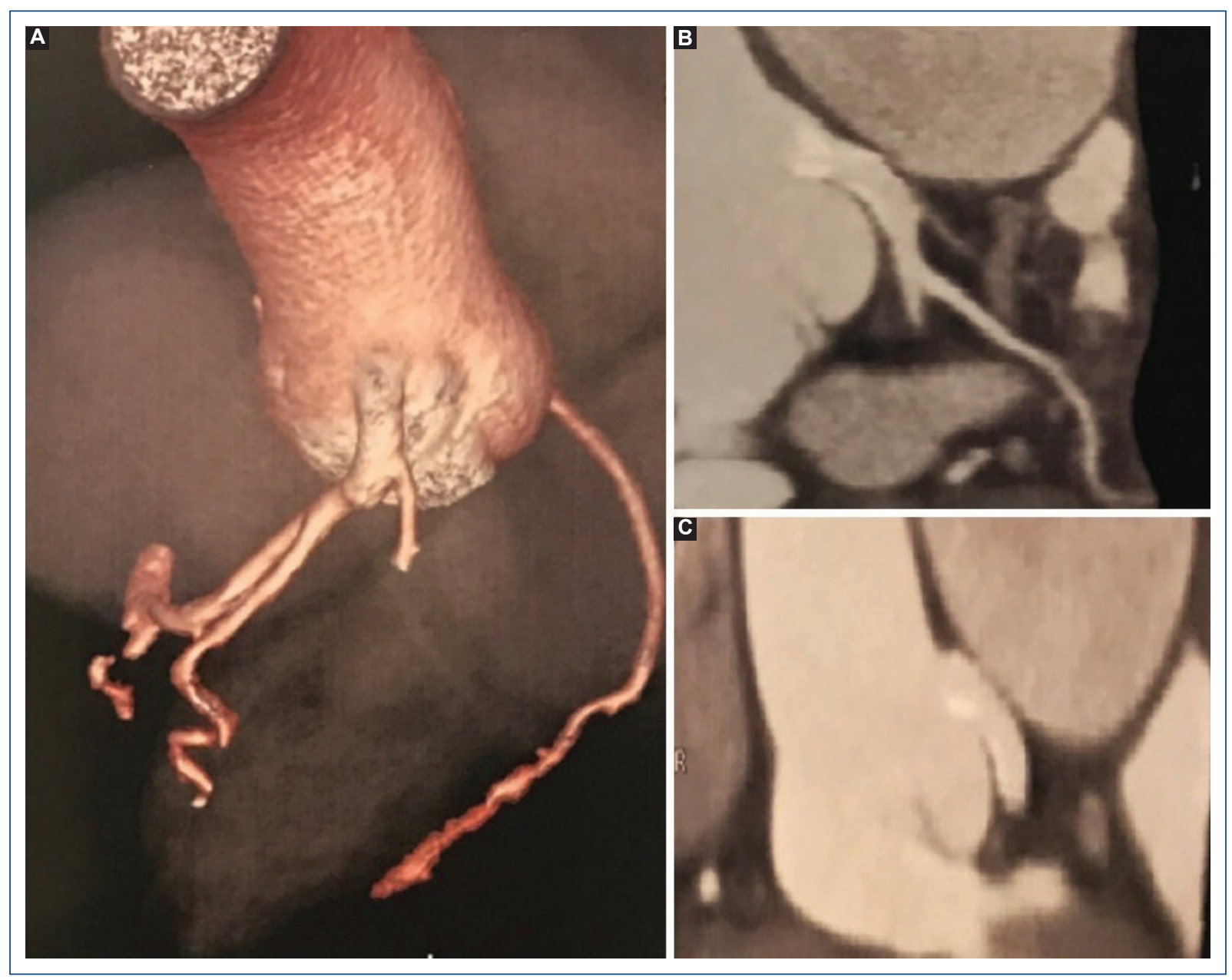

Figura 2. A: angiotomografía de tórax, reconstrucción tridimensional: se observa conservado el calibre del stent colocado en el tronco de la arteria coronaria izquierda, sin signos de reestenosis o compresión. B: angiotomografia de tórax: se presentan dos cortes en los que se visualiza la ausencia de complicaciones relacionadas con el stent implantado; el tronco de la coronaria izquierda muestra un trayecto anómalo debido a la dilatación de la arteria pulmonar.

en el riesgo hemorrágico del paciente intervenido, con uso de stents convencionales en aquéllos con alguna predisposición al sangrado o con necesidad de tratamiento anticoagulante por otro motivo, para reducir al mínimo los tiempos del doble tratamiento antiagregante ${ }^{6}$.

Existen escasos datos con respecto a la evolución y la tasa de complicaciones de estos procedimientos a largo plazo. En el caso informado, ante la ausencia de síntomas referidos por la paciente luego del procedimiento, se realizó cada año una angiotomografía de tórax, la última tres años después del implante del stent, sin hallar evidencia de reestenosis ni compresión extrínseca (Fig. 2).

A modo de conclusión, la compresión extrínseca del tronco de la coronaria izquierda por la dilatación de la arteria pulmonar es una causa cada vez más reconocida de angina u otras complicaciones más graves en pacientes con diagnóstico de hipertensión pulmonar. Su identificación debe ser precisa a través de la sospecha clínica y la utilización de los métodos complementarios ya comentados. El tratamiento de primera línea es el endovascular, considerado el alto riesgo quirúrgico de estos pacientes. El seguimiento realizado a tres años desde el procedimiento sin evidenciar complicaciones tiene la intención de establecer un aporte en relación con la seguridad de esta terapéutica a largo plazo.

\section{Conflicto de intereses}

Ninguno. 


\section{Financiamiento}

La presente investigación no ha recibido ninguna beca específica de agencias de los sectores público, comercial, o sin ánimo de lucro.

\section{Responsabilidades éticas}

Protección de personas y animales. Los autores declaran que para esta investigación no se han realizado experimentos en seres humanos ni en animales.

Confidencialidad de los datos. Los autores declaran que han seguido los protocolos de su centro de trabajo sobre la publicación de datos de pacientes.

Derecho a la privacidad y consentimiento informado. Los autores han obtenido el consentimiento informado de los pacientes o sujetos referidos en el artículo. Este documento obra en poder del autor de correspondencia.

\section{Bibliografía}

1. Lee S-E, An HY, Im JH, Sung JM, Cho I-J, Shim CY, et al. Screening of mechanical complications of dilated pulmonary artery related to the risk for sudden cardiac death in patients with pulmonary arterial hypertension by transthoracic echocardiography. J Am Soc Echocardiogr. 2016; 29(6):561-6

2. Mesquita SMF, Castro CRP, Ikari NM, Oliveira SA, Lopes AA. Likelihood of left main coronary artery compression based on pulmonary trunk diameter in patients with pulmonary hypertension. Am $\mathrm{J}$ Med. 2004;15;116(6):369-74.

3. Seabra LF, Ribeiro HB, de Barros e Silva PGM, Rodrigues MJ, Spadaro AG, Conejo $F$, et al. Left main ostial compression in a patient with pulmonary hypertension: dynamic findings by IVUS. Am J Case Rep. 2015;16:899-903.

4. Bhoil R, Sood S, Bhoil S, Chamail A, Sood RG. Extrinsic compression of left main coronary artery due to dilated pulmonary trunk resulting in ischaemic symptoms. Acute Card Care. 2016;18(1):23-4.

5. Alfonso F, Rivero F. Left main coronary artery compression in patients with pulmonary arterial hypertension. J Am Coll Cardiol. 2017; 69(23):2818-20

6. Galiè N, Saia F, Palazzini M, Manes A, Russo V, Bacchi Reggiani ML, et al. Left main coronary artery compression in patients with pulmonary arterial hypertension and angina. J Am Coll Cardiol. 2017;69(23):2808-17.

7. Demerouti EA, Manginas AN, Athanassopoulos GD, Karatasakis GT. Complications leading to sudden cardiac death in pulmonary arterial hypertension. Respir Care. 2013;58(7):1246-54.

8. Caldera AE, Cruz-González I, Bezerra HG, Cury RC, Palacios IF, Cockrill BA, et al. Endovascular therapy for left main compression syndrome. Case report and literature review. Chest. 2009;135(6):1648-50. 